PROCEEDINGS OF THE AMERICAN MATHEMATICAL SOCIETY

Volume 127, Number 3, March 1999, Pages 647-655

S 0002-9939(99)04531-1

\title{
DERIVED TUBULAR STRONGLY SIMPLY CONNECTED ALGEBRAS
}

\author{
M. BAROT AND J. A. DE LA PEÑA \\ (Communicated by Ken Goodearl)
}

\begin{abstract}
Let $A$ be a finite dimensional algebra over an algebraically closed field $k$. Assume $A=k Q / I$ for a connected quiver $Q$ and an admissible ideal $I$ of $k Q$. We study algebras $A$ which are derived equivalent to tubular algebras. If $A$ is strongly simply connected and $Q$ has more than six vertices, then $A$ is derived tubular if and only if (i) the homological quadratic form $\chi_{A}$ is a non-negative of corank two and (ii) no vector of $\chi_{A}^{-1}(1)$ is orthogonal (with respect tho the homological bilinear form) to the radical of $\chi_{A}$.
\end{abstract}

Finite dimensional algebras over an algebraically closed field $k$ are divided into two classes. On one hand, we have the tame algebras for which almost all indecomposable modules of each fixed dimension occur in a finite number of one-parametric families; on the other hand, the wild algebras for which the classification of indecomposable modules contains the classification of pairs of matrices under simultaneous conjugations. Among tame algebras, tubular algebras form a well-understood and important class [15]. The aim of this work is to study algebras which are derived equivalent to tubular algebras. We shall apply our results to obtain a new characterization of polynomial growth algebras.

Two $k$-algebras $A_{1}$ and $A_{2}$ are said to be derived equivalent if their derived categories $\mathrm{D}^{\mathrm{b}}\left(A_{1}\right.$-mod $)$ and $\mathrm{D}^{\mathrm{b}}\left(A_{2}\right.$-mod $)$ are equivalent as triangulated categories; see [10]. If $A_{1}$ and $A_{2}$ are derived equivalent and $A_{1}$ is tubular, we say that $A_{2}$ is a derived tubular algebra. In [4], a characterization of derived tubular algebras of finite representation type was given; the characterization uses properties of the homological form and the Tits form of the algebra. In this work we extend those results to certain classes of tame algebras.

If the algebra $A$ is of finite global dimension, then the homological bilinear form of $A$ is defined by $\langle\underline{\operatorname{dim}} M, \underline{\operatorname{dim}} N\rangle_{A}=\sum_{i=0}^{\infty}(-1)^{i} \operatorname{dim}_{k} \operatorname{Ext}_{A}^{i}(M, N)$, where $\underline{\operatorname{dim}} M$ denotes the class of an $A$-module $M$ in the Grothendieck group $\mathrm{K}_{\circ}(A)$ of $A$. The corresponding quadratic form is denoted by $\chi_{A}$, whereas the Tits form $\mathrm{q}_{A}$ is the "truncated version", defined for a semisimple module $S$ as $\mathrm{q}_{A}(\underline{\operatorname{dim}} S)=$ $\sum_{i=0}^{2}(-1)^{i} \operatorname{dim}_{k} \operatorname{Ext}^{i}(S, S)$. The radical $\mathrm{rad}_{A}$ is the subgroup of $\mathrm{K}_{\circ}(A)$ of all vectors $v$ such that $\mathrm{q}_{A}(v+?)=\mathrm{q}_{A}(v)+\mathrm{q}_{A}(?)$. The corank of $\mathrm{q}_{A}$ is the rank of $\operatorname{rad} \mathrm{q}_{A}$.

Let $Q$ be a connected quiver and $I$ an admissible ideal of $k Q$ such that $A=k Q / I$ is a finite dimensional $k$-algebra. We recall from [17] that $A$ is said to be strongly

Received by the editors December 2, 1996 and, in revised form, June 12, 1997.

1991 Mathematics Subject Classification. Primary 16G10, 16G60, 18E30.

This work was partially supported by CONACYT and DGAPA, UNAM.

(C)1999 American Mathematical Society 
simply connected if $Q$ has no oriented cycle and for every convex subcategory $B$ of $A$, the first Hochschild cohomology group $\mathrm{H}^{1}(B, B)$ vanishes. The main results of this work are the following.

Theorem A. Let $Q$ be a connected quiver with more than six vertices and $I$ an admissible ideal of $k Q$ such that $A=k Q / I$ is strongly simply connected. Then $A$ is derived tubular if and only if A satisfies the following two conditions.

(i) The homological quadratic form $\chi_{A}$ is non-negative and has corank two,

(ii) No vector of $\chi_{A}^{-1}(1)$ is orthogonal (with respect to the homological bilinear form) to the whole radical of $\chi_{A}$.

Theorem B. Let $Q$ be a connected quiver with more than six vertices and $I$ an admissible ideal of $k Q$ such that $A=k Q / I$ is strongly simply connected. Then $A$ is tubular if and only if $A$ satisfies the following three conditions.

(i) The Tits form $q_{A}$ is non-negative and has corank two,

(ii) No vector of $\mathrm{q}_{A}^{-1}(1)$ is orthogonal (with respect to the homological bilinear form) to the whole radical of $q_{A}$.

(iii) There exists a positive sincere radical vector of $q_{A}$.

In section 2 we shall provide a complete list of derived tubular algebras whose underlying quivers have six vertices. This is relevant since for this number of vertices the above characterizations fail.

For a tame algebra $A$, and $d \in \mathbb{N}$ denote by $\mu_{A}(d)$ the minimal number of oneparametric families of indecomposable $d$-dimensional $A$-modules. We say that $A$ is of polynomial growth if $\mu_{A}(d) \leq d^{m}$ for some $m \in \mathbb{N}$ and all $d \in \mathbb{N}$. In [13], strongly simply connected polynomial growth algebras are characterized by properties of $\mathrm{q}_{A}$. From those results and Theorem B we obtain the following characterization.

Theorem C. Let $A$ be a strongly simply connected algebra. Then $A$ is of polynomial growth if and only if $\mathrm{q}_{A}$ is weakly non-negative and any full convex subcategory $B$ of $A$ with non-negative Tits form $q_{B}$ of corank two and a sincere, positive radical vector of $q_{B}$ satisfies the following condition: either $B$ is isomorphic to $\mathbf{A}_{\rho}, \mathbf{B}_{\rho}$ or $\mathbf{B}_{\rho}^{\text {op }}$ in list 2.4, or $B$ has more than six points and then no vector in $\mathrm{q}_{B}^{-1}(1)$ is orthogonal (with respect to the homological bilinear form) to the whole radical of $\mathrm{q}_{B}$.

Proofs of the results are given in section 4 after some required preparation in section 3. For a general discussion on quadratic forms and representation theory see for example [14].

\section{Fundamental CONCEPTS}

Throughout the article, $k$ denotes a fixed, algebraically closed field. A spectroid is a $k$-category with finite dimensional morphism spaces and pairwise nonisomorphic objects (called points) whose endomorphism algebras are local. Basic algebras over $k$ stand in a one-to-one correspondence with finite spectroids, $A \longleftrightarrow \mathcal{S}_{A}$ [9]. The category $A$-mod of finite-dimensional left-modules over $A$, is equivalent to the category $\mathcal{S}_{A}$-mod of covariant finitely generated functors (also called left $\mathcal{S}_{A}$-modules) $\mathcal{S}_{A} \rightarrow k$-mod. Since only left $\mathcal{S}$-modules will be considered, the specification "left" will be suppressed. The category of finite dimensional $\mathcal{S}$ modules is denoted by $\mathcal{S}$-mod. For each class of indecomposable $\mathcal{S}$-modules choose 
a representative. Then the full subcategory of $\mathcal{S}$-mod given by those representatives is a spectroid which will be denoted by $\mathcal{S}$-ind.

A spectroid $\mathcal{S}$ is finite if it has only finitely many points, it is directed if there does not exist a cycle of non-zero morphisms in $\mathcal{S}$, and it is connected if the points of $\mathcal{S}$ cannot be divided into two non-empty sets with only zero morphisms between them.

If a spectroid $\mathcal{S}$ is directed the associated quiver of $\mathcal{S}$ induces a partial order on the objects of $\mathcal{S}$. The maximal points correspond to the sinks whereas the minimal points correspond to the sources.

1.1. Let $\mathcal{S}$ be a finite and directed spectroid. Then its repetitive spectroid $\widehat{\mathcal{S}}$ is a spectroid having as object-set $\mathcal{S} \times \mathbb{Z}($ instead of $\mathrm{Ob} \mathcal{S}$ we just write $\mathcal{S}$, the points are denoted by $s[i], s \in \mathcal{S}, i \in \mathbb{Z})$ and non-zero morphism-spaces $\widehat{\mathcal{S}}(r[i], s[i])=$ $\mathcal{S}(r, s) \times\{i\}$ and $\widehat{\mathcal{S}}(r[i], s[i-1])=\mathrm{D} \mathcal{S}(s, r) \times\{i\}$.

The bijection ?[j] from the points of $\widehat{\mathcal{S}}$ to itself, $(s[i])[j]:=s[i+j]$ extends for any $j \in \mathbb{Z}$ in a natural way to an automorphism of $\widehat{\mathcal{S}}$, which again will be called ?[j].

1.2. The source-extension $\mathcal{S}$ (denoted by $\mathcal{S}_{\circ}[M]$ ) of a spectroid $\mathcal{S}_{\circ}$ by a $\mathcal{S}_{\circ}$ module $M$ is a spectroid having as points those of $\mathcal{S}_{\circ}$, an additional point $\alpha_{M}$ and morphism-spaces given by $\left.\mathcal{S}\right|_{\mathcal{S}_{\circ}}=\mathcal{S}_{\circ}, \mathcal{S}\left(\alpha_{M}\right.$, ?) $\left.\right|_{\mathcal{S}_{\circ}}=M$ (as left $\mathcal{S}_{\circ}$-modules), $\mathcal{S}\left(?, \alpha_{M}\right)=0$ and $\mathcal{S}\left(\alpha_{M}, \alpha_{M}\right)=k$. The sink-extension (denoted by $[M] \mathcal{S}_{\circ}$ ) of $\mathcal{S}$ by $M$ is defined dually. If $\mathcal{S}$ is a finite spectroid, $\omega$ a maximal point of $\mathcal{S}$ and if $I_{\alpha}$ denotes the injective indecomposable $\mathcal{S}$-module corresponding to $\omega$, then the spectroid $\rho_{\omega}^{+} \mathcal{S}$ which is obtained from $\mathcal{S}$ by deleting $\omega$ (i.e. taking the full subspectroid of $\mathcal{S}$ given by all points $\beta \neq \omega$ ) and then building the source-extension in $I_{\omega} / \operatorname{soc} I_{\omega}$ is called the source-reflection of $\mathcal{S}$ in $\omega$.

Dually for a minimal point $\alpha$ in $\mathcal{S}$ we define the sink-reflection of $\mathcal{S}$ in $\alpha$ and denote it by $\rho_{\alpha}^{-} \mathcal{S}$.

The unique point in $\rho_{\omega}^{+} \mathcal{S} \backslash \mathcal{S}_{\circ}$ will be denoted by $\omega[1]$, in the first case, and the unique point in $\rho_{\alpha}^{-} \mathcal{S} \backslash \mathcal{S}_{\circ}^{\prime}$ by $\alpha[-1]$. Two spectriods are called reflection-equivalent if one is obtained from the other by a sequence of source- and sink-reflections.

1.3. It is sometimes useful to mark a point $p$ of a spectroid $\mathcal{S}$ and to consider pointed spectroids. Let $A:=\overrightarrow{\mathbb{A}_{n}}$ denote the linearily ordered spectroid with $n$ points, $P$ the unique projective-injective indecomposable and $T$ a tilting $A$-module. If we vary $n$ and $T$ we obtain simple but important examples of pointed finite spectroids $(\operatorname{End}(T), P)$, where $\mathcal{T}=\operatorname{End}(T)$ is the spectroid whose points $T_{i}, i=$ $1, \ldots, n$ are chosen representatives of the isomorphism classes of the indecomposable direct summands of $T$ and whose morphism spaces are $\mathcal{T}\left(T_{i}, T_{j}\right)=\operatorname{Hom}\left(T_{i}, T_{j}\right)$. These pointed spectroids are called branches. For a more descriptive definition see for example [15].

Now let $\dot{\mathcal{R}}=(\mathcal{R}, r)$ and $\dot{\mathcal{S}}=(\mathcal{S}, s)$ be two pointed spectroids. The glueing of $\mathcal{R}$ with $\mathcal{S}$ is a spectroid, defined as the fibre-product of $\iota_{r}:\{\odot\} \longrightarrow \mathcal{R}, \odot \mapsto r$ and $\iota_{s}:\{\odot\} \longrightarrow \mathcal{S}, \odot \mapsto s$.

For a spectroid $\mathcal{S}$ and an indecomposable $\mathcal{S}$-module $M$, the branch-sourceextension $\mathcal{S}[M, \dot{\mathcal{B}}]$ of $\mathcal{S}$ by a branch $\dot{\mathcal{B}}$ in $M$ is the glueing of $\left(\mathcal{S}[M], \alpha_{M}\right)$ with $\dot{\mathcal{B}}$, and dually the branch-sink-extension $[\dot{\mathcal{B}}, M] \mathcal{S}$ is the glueing of $\left([M] \mathcal{S}, \omega_{M}\right)$ with $\dot{\mathcal{B}}$. 
Let $\mathcal{S}$ be a finite spectroid, $s$ an extremal point of $\mathcal{S}$ and $\mathcal{R}$ a full and convex subspectroid of $\mathcal{S}$. We then say that the reflection of $\mathcal{S}$ in $s$ avoids $\mathcal{R}$ if $s$ does not belong to $\mathcal{R}$. In particular $\mathcal{R}$ is preserved as a full and convex subspectroid under the reflection. A sequence of reflections avoids $\mathcal{R}$ if each reflection of the sequence avoids $\mathcal{R}$. Any finite spectroid which can be obtained from a branchsource-extension of a spectroid $\mathcal{S}_{\circ}$ by a sequence of reflections avoiding $\mathcal{S}_{\circ}$ is called a branch-enlargement of $\mathcal{S}_{\circ}$.

1.4. Let $\mathcal{C}$ be a critical spectroid (for a definition we refer to [15]). If $\left(\mathcal{T}_{\lambda}\right)_{\lambda \in \mathbb{P}_{1} k}$ is the tubular family in $\mathcal{C}$-ind, then the function $t_{\mathcal{C}}: \mathbb{P}_{1} k \longrightarrow \mathbb{N}, \lambda \mapsto \operatorname{rk}\left(\mathcal{T}_{\lambda}\right)$ is called the tubular-type-function, where $\operatorname{rk}(\mathcal{T})$ denote the rank of $\mathcal{T}_{\lambda}$, that is, the number of modules in the mouth of $\mathcal{T}_{\lambda}$. Since almost everywhere $t_{\mathcal{C}}$ takes the value one, usually one writes only the tubular type $\left(t_{\mathcal{C}}\left(\lambda_{1}\right), \ldots, t_{\mathcal{C}}\left(\lambda_{p}\right)\right)$ of $\mathcal{C}$, where $\mathcal{T}_{\lambda_{1}}, \ldots, \mathcal{T}_{\lambda_{p}}$ are the non-homogeneous tubes in $\mathcal{C}$-ind [15].

A 1-regular branch-source-extension of $\mathcal{C}$ is a branch-source-extension of $\mathcal{C}$ by a regular, indecomposable module of colength 1 . The colength of a module in an arbitrary tube is the position in the coray through $M$. The tubular-typefunction of $\mathcal{C}[M, \dot{\mathcal{B}}]$ is defined by $t_{\mathcal{C}[M, \dot{\mathcal{B}}]}(\mu)=t_{\mathcal{C}}(\mu)+|\mathcal{B}|$, if $M$ belongs to $\mathcal{T}_{\mu}$, and $t_{\mathcal{C}[M, \dot{\mathcal{B}}]}(\lambda)=t_{\mathcal{C}}(\lambda)$ for all $\lambda \neq \mu$. Let $M_{1}, \ldots, M_{n}$ be pairwise distinct, indecomposable, regular $\mathcal{C}$-modules, all of colength one and $\dot{\mathcal{B}}_{1}, \ldots, \dot{\mathcal{B}}_{n}$ branches. The spectroid $\mathcal{C}\left[M_{1}, \dot{\mathcal{B}}_{1}\right] \cdots\left[M_{m}, \dot{\mathcal{B}}_{m}\right]$ will still be called a 1-regular branch-source-extension of $\mathcal{C}$ and its tubular type function is defined iteratively.

The 1-regular branch-source-extensions of a critical spectroid $\mathcal{C}$ have been extensively studied $[3,12,15]$. The distinction of tame and wild ones can be easily formulated by means of the tubular type; namely, the spectroid $\mathcal{S}=\mathcal{C}\left[M_{1}, \dot{\mathcal{B}}_{1}\right] \cdots\left[M_{m}, \dot{\mathcal{B}}_{m}\right]$ is tame if and only if the star $\Gamma_{\left(t_{1}, \ldots t_{p}\right)}$ associated to the tubular type $\left(t_{1}, \ldots, t_{p}\right)$ of $\mathcal{S}$ is Dynkin or extended Dynkin [12,15]. In the first case $\mathcal{S}$ is called domestic tubular, whereas in the latter tubular. The expressions "derived equivalent to a tubular (resp. domestic tubular) spectroid of tubular type $\left(t_{1}, \ldots, t_{p}\right)$ " will be shortened to derived tubular (resp. derived domestic tubular) of type $\left(t_{1}, \ldots, t_{p}\right)$.

1.5. From [5], we recall that a spectroid $\mathcal{S}$ satisfies the separation condition if for each point $s$ of $\mathcal{S}$, two different direct summands of $\operatorname{rad} \mathrm{P}_{s}$ lie in different connected components of the spectroid $\mathcal{S} \backslash s^{\triangleleft}$, where $s^{\triangleleft}$ is the full subspectroid of $\mathcal{S}$ given by the start points of all paths ending at $s$.

By [17], a spectroid $\mathcal{S}$ is strongly simply connected if and only if each full and convex subspectroid of $\mathcal{S}$ satisfies the separation condition. If, in addition, $\mathcal{S}$ is of finite representation type, then by [8], $\mathcal{S}$ is strongly simply connected if and only if $\mathcal{S}$ itself satisfies the separation condition.

\section{SpeCtroids With SIX POINTS}

2.1. A finite spectroid $\mathcal{S}$ is called 2-tubular if it is isomorphic to a sourceextension $\mathcal{D}[M]$, where $\mathcal{D}$ is a domestic tubular spectroid of tubular type $(2,2, s)$, for some $s \geq 2$, and $M$ is an indecomposable regular $\mathcal{D}$-module of colength two lying in a tube $\mathcal{T}_{\lambda}$ with $t_{\mathcal{D}}(\lambda)=s$. Important examples of 2-tubular spectroids are the strongly simply connected pg-critical spectroids as defined in [18]. 
It is known that tubular and 2-tubular spectroids have "similar" quadratic forms. However, if the considered spectroids have more than six points (the smallest tubular and 2-tubular spectroids have six points), then there is a distinction of their quadratic forms.

We recall from the introduction that $\chi_{\mathcal{S}}$ denotes the associated quadratic form to the homological bilinear form $\langle ?,-\rangle_{\mathcal{S}}$. Furthermore we define for any subset $U \subset \mathrm{K}_{\circ}(\mathcal{S})$ its right orthogonal group $U^{\perp}=\left\{v \in \mathrm{K}_{\circ}(\mathcal{S}) \mid \forall u \in U,\langle u, v\rangle_{\mathcal{S}}=0\right\}$.

2.2. Distinction Lemma $([4])$. Let $\mathcal{S}$ be a finite spectroid with more than six points.

(a) If $\mathcal{S}$ is derived tubular, then $\chi_{\mathcal{S}}{ }^{-1}(1) \cap\left(\operatorname{rad} \chi_{\mathcal{S}}\right)^{\perp}=\emptyset$,

(b) if $\mathcal{S}$ is derived 2-tubular, then $\chi_{\mathcal{S}}{ }^{-1}(1) \cap\left(\operatorname{rad} \chi_{\mathcal{S}}\right)^{\perp} \neq \emptyset$.

Proof. The proof is roughly sketched, for more details we refer to [4].

For (a) we assume that there exists a vector $v$ in $\mathcal{S}^{-1}(1) \cap(\operatorname{rad} \mathcal{S})^{\perp}$. Let $w$ be a sincere positive, radical vector with $\left\langle h_{\circ}, w\right\rangle_{\mathcal{S}}=-\left\langle h_{\infty}, w\right\rangle_{\mathcal{S}}$, where $h_{\circ}$ and $h_{\infty}$ denote the positive radical vectors corresponding to the two critical, full and convex subspectroids of $\mathcal{S}$. For a sufficiently large natural number $N$, the vector $v^{\prime}=v+$ $N w$ is then sincere, positive and satisfies $\chi_{\mathcal{S}}\left(v^{\prime}\right)=1$ and $\left\langle h_{\circ}, v^{\prime}\right\rangle_{\mathcal{S}}=-\left\langle h_{\infty}, v^{\prime}\right\rangle_{\mathcal{S}}=$ $N\left\langle h_{\circ}, w\right\rangle_{\mathcal{S}}$. The latter implies that $\chi_{\mathcal{S}}\left(v^{\prime}\right)=0$, a fact, which contradicts the above statement.

In order to prove (b), we set $\mathcal{S}=\mathcal{D}[M]$ as in the definition above. Let $N$ be the indecomposable, regular $\mathcal{D}$-module of colength one, which lies on the same coray as $M$. We now show first that $\Phi_{\mathcal{S}} \underline{\operatorname{dim}} N+\underline{\operatorname{dim}} N=\underline{\operatorname{dim}} \mathrm{P}_{\alpha}-\underline{\operatorname{dim}} \mathrm{S}_{\alpha}$ where $\Phi_{\mathcal{S}}$ denotes the Coxeter transformation of $\mathcal{S}$ and $\alpha=\alpha_{M}$. From this it follows that $u:=\Phi_{\mathcal{S}}^{2} \underline{\operatorname{dim}} N+\underline{\operatorname{dim}} \mathrm{S}_{\alpha}$ belongs to $\left(\operatorname{rad} \chi_{\mathcal{S}}\right)^{\perp}$. Finally we verify that $\chi_{\mathcal{S}}(u)=1$ also holds.

2.3. The condition on the number of points in 2.2 is necessary as shown by the following considerations.

Let $\mathcal{D}[M]$ be a 2 -tubular spectroid with six points. The tubular type of $\mathcal{D}$ is then $(2,2,2)$ and therefore the tube to which the module $M$ belongs is stable and of rank two. Since $M$ has colength two, it follows that the dimension vector of $M$ is the same as for any indecomposable, regular module $M^{\prime}$, of colength one lying in a homogeneous tube.

Hence the Cartan matrices of the spectroids $\mathcal{D}[M]$ and $\mathcal{D}\left[M^{\prime}\right]$ are the same and so are the homological bilinear forms. Since both spectroids have global dimension two, the homological quadratic forms such as the Tits forms are all indistinguishable.

2.4. Fortunately, in the situation considered in 2.3 it is easy to determine all spectroids explicitly. First in Figure 1, part I, we obtain the list of all tubular spectroids with six points, where every picture represents a family of spectroids (the index $\rho$ runs over a cofinite subset of the field $k$ and hits each isoclass of algebras only finitely many times). This list was also calculated in [16].

Using that a derived tubular spectroid is always reflection-equivalent to a tubular spectroid [1], we obtain furthermore the spectroids shown in Figure 1, part II.

Figure 1, part I and part II, exhibits all derived tubular spectroids with six points. Only the first three families, namely $\left(\mathbf{A}_{\rho}\right),\left(\mathbf{B}_{\rho}\right)$ and $\left(\mathbf{B}_{\rho}^{\mathrm{op}}\right)$ are strongly simply connected. 


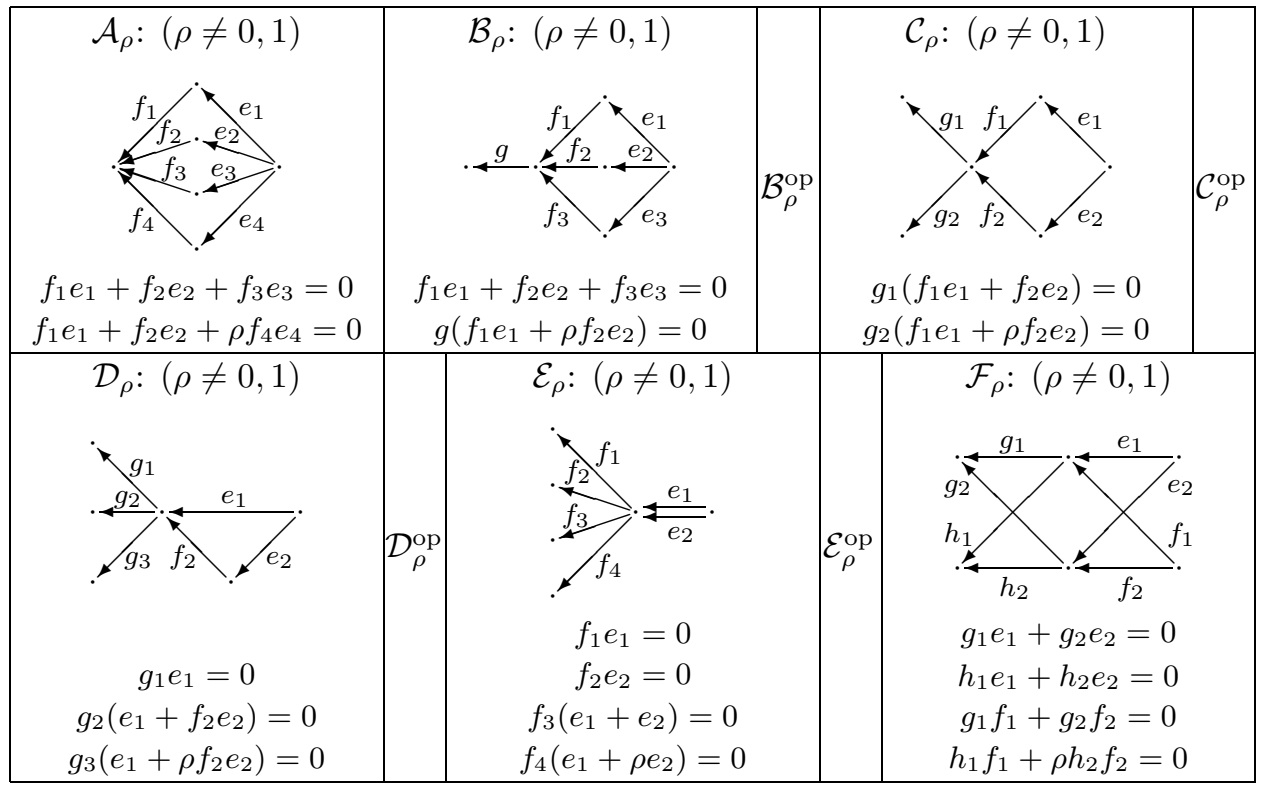

Figure 1, PART I

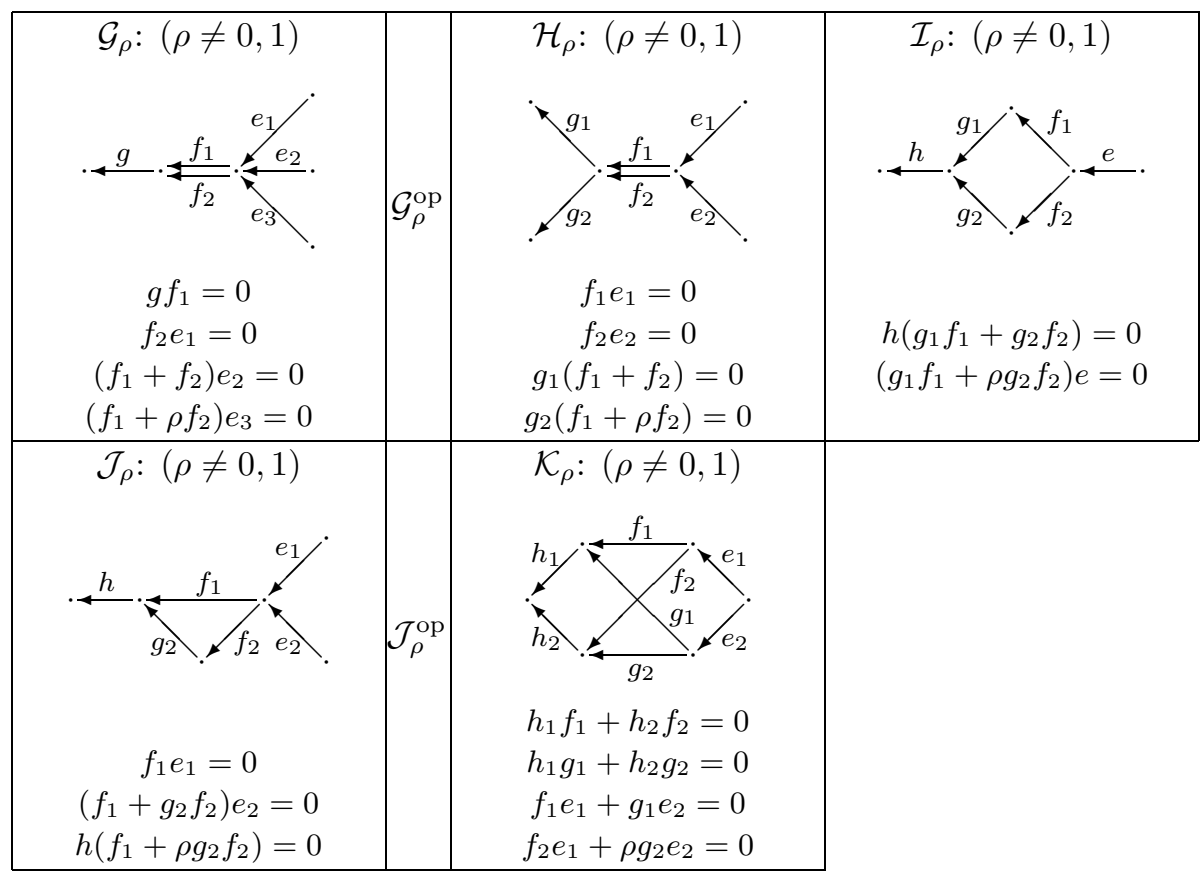

Figure 1, PART II 


\section{TOOLS}

3.1. For the proof of the theorems we proceed by reduction deleting special extremal points. In this section we discuss this method.

Proposition. Let $\mathcal{S}$ be a finite and directed spectroid with a non-negative (respectively positive) homological quadratic form, then any full subspectroid $\mathcal{S} \circ$ of $\mathcal{S}$ has again a non-negative (respectively positive) homological quadratic form.

Proof. The proof is done by induction on the number of points of $\mathcal{S}$ which do not belong to $\mathcal{S}_{\circ}$.

Let $s$ be a point of $\mathcal{S}$ and set $\mathcal{S}_{\circ}=\mathcal{S} \backslash\{s\}$. Then define $s^{\triangleleft}$ as in 1.5. Let $s_{1}$ be minimal in $s^{\triangleleft}, s_{2}$ be minimal in $s^{\triangleleft} \backslash\left\{s_{1}\right\}, s_{3}$ be minimal in $s^{\triangleleft} \backslash\left\{s_{1}, s_{2}\right\}$, and so on, until we end up with $s^{\triangleleft} \backslash\left\{s_{1}, \ldots, s_{q}\right\}=\{s\}$. Then set $\mathcal{R}=\rho_{s_{q}}^{-} \cdots \rho_{s_{1}}^{-} \mathcal{S}$.

Observe that reflections provide isomorphisms between the Grothendieck groups preserving the homological bilinear form. Therefore $\chi_{\mathcal{R}}$ is non-negative (respectively positive) again. The point $s$ is minimal in $\mathcal{R}$, thus $\mathcal{R}_{\circ}=\mathcal{R} \backslash\{s\}$ is convex in $\mathcal{R}$. Hence $\chi_{\mathcal{R}}$ is non-negative (respectively positive) again. Now the assertion follows again by the observation above observing that $\mathcal{S}_{\circ}$ is isomorphic to $\rho_{s_{1}[-1]}^{+} \cdots \rho_{s_{q}[-1]}^{+} \mathcal{R}_{\circ}$.

Observe that $\chi_{\mathcal{S}_{\circ}}$ is not always the restriction of $\chi_{\mathcal{S}}$.

3.2. Lemma. Let $\mathcal{S}$ be a directed spectroid, which satisfies the following two conditions.

(i) The homological quadratic form $\chi_{\mathcal{S}}$ is non-negative and has corank two and

(ii) $\chi_{\mathcal{S}}{ }^{-1}(1) \cap\left(\operatorname{rad} \chi_{\mathcal{S}}\right)^{\perp}=\emptyset$.

Furthermore, let $s$ be a point of $\mathcal{S}$. Then the homological quadratic form $\chi_{\mathcal{S}}$ is non-negative of corank one, where $\mathcal{S}_{\circ}$ denotes the spectroid $\mathcal{S} \backslash\{s\}$.

Proof. As in the lemma above we can restrict, without loss of generality, to the case where $s$ is maximal. The quadratic form $\chi_{\mathcal{S}_{0}}$ is then just the restriction of $\chi_{\mathcal{S}}$ to $\mathcal{S}_{\circ}$. Since the corank of $\chi_{\mathcal{S}}$ is two, it is easy to find a radical vector $v$ such that $v(s)=0$. This shows that the corank of $\chi_{\mathcal{S}_{\circ}}$ is at least one. If there are two linearily independent radical vectors $w_{1}$ and $w_{2}$ of $\chi_{\mathcal{S}}$, then this would imply, that the dimension vector of the indecomposable projective $\mathcal{S}$-module corresponding to $s$ belongs to $\operatorname{rad} \chi_{\mathcal{S}}{ }^{\perp}$, and hence also to $\chi_{\mathcal{S}}{ }^{-1}(1) \cap\left(\operatorname{rad} \chi_{\mathcal{S}}\right)^{\perp}$, in contradiction to the condition (ii).

3.3. The basic piece in our tool box is the following.

Proposition ([4]). If $\mathcal{D}$ is a derived domestic tubular spectroid and $M$ an indecomposable $\mathcal{D}$-module, such that $\chi_{\mathcal{D}[M]}$ is non-negative, then there exists a hereditary critical spectroid $\mathcal{H}$, an indecomposable regular $\mathcal{H}$-module $N$ and an equivalence between $\mathrm{D}^{\mathrm{b}}\left(\mathcal{D}\right.$-mod) and $\mathrm{D}^{\mathrm{b}}(\mathcal{H}$-mod) as triangulated categories which extend to an equivalence between $\mathrm{D}^{\mathrm{b}}\left(\mathcal{D}[M]\right.$-mod) and $\mathrm{D}^{\mathrm{b}}(\mathcal{H}[N]$-mod) as triangulated categories.

Proof. For more details we refer to [4]. First, show that the module $M$ considered as $\widehat{\mathcal{D}}$-module, lies in one of the tubular families of $\widehat{\mathcal{D}}$-ind. Then one verifies that the support of $M$ in $\widehat{\mathcal{D}}$ is a branch-enlargement $\mathcal{E}$ of a special shape of a critical spectroid. Finally we prove, that $\mathcal{E}[M]$ can be transformed by a sequence of tiltings and cotiltings into a source-extension of a critical, hereditary spectroid $\mathcal{H}$ by an indecomposable, regular module $N$. 
3.4. Proposition. Let $\mathcal{S}$ be a finite, connected and strongly simply connected spectroid. Then $\mathcal{S}$ is derived domestic tubular if and only if $\chi_{\mathcal{S}}$ is non-negative and has corank one.

Proof. Let $\mathcal{S}$ be a finite, connected and strongly simply connected spectroid with a non-negative homological quadratic form of corank one. If $\mathcal{S}$ is of finite representation type, then the result follows from [3]. In the remaining case we show that, according to [7], there exists a full and convex subspectroid $\mathcal{R}$ of $\mathcal{S}$, which is critical. If $\mathcal{R}=\mathcal{S}$ we are done. Otherwise we may find an extremal point $s$ in $\mathcal{S}$ such that $\mathcal{S}_{\circ}=\mathcal{S} \backslash\{s\}$ is still connected and contains $\mathcal{R}$. By induction $\mathcal{S}_{\circ}$ is derived domestic tubular. If $s$ is minimal, then $\operatorname{rad}_{s}$ is indecomposable and therefore by Proposition 3.3, the result follows. If on the other hand $s$ is maximal, then we apply the same argument to $\rho_{s}^{-} \mathcal{S}$, where we show that the opposite of a strongly simply connected spectroid is strongly simply connected again [17].

\section{Proof of the Results}

4.1. Proof of Theorem A. The necessity of the conditions (i) and (ii) follows from 2.2 and $[15]$.

For the sufficiency we choose an extremal point $s$ of $\mathcal{S}$ such that $\mathcal{S}_{\circ}:=\mathcal{S} \backslash\{s\}$ is connected. By 3.1 the homological quadratic form of $\mathcal{S}_{\circ}$ is non-negative and by 3.2 it has corank one. Since $\mathcal{S}_{\circ}$ is full and convex in $\mathcal{S}$, the spectroid $\mathcal{S}_{\circ}$ is strongly simply connected again, and therefore 3.4 implies, that $\mathcal{S}_{\circ}$ is derived domestic tubular. If the point $s$ is maximal in $\mathcal{S}$ we work with the opposite spectroid $\mathcal{S}^{\mathrm{op}}$.

So suppose, that the point $s$ is minimal in $\mathcal{S}$. The module $\operatorname{rad}_{s}$ is indecomposable and hence 3.3 may be applied, in order to obtain, that $\mathcal{S}$ is derived equivalent to a source-extension of a hereditary spectroid $\mathcal{H}$ by an indecomposable regular $\mathcal{H}$-module $N$. By [12] the non-negativity of the quadratic form of $\mathcal{H}[N]$ implies that $\mathcal{H}[N]$ is either domestic tubular, tubular or 2-tubular. The first is excluded by the corank of the quadratic form and the latter by condition (ii) and 2.2.

4.2. Corollory. Let $\mathcal{S}$ be a strongly simply connected spectroid with more than six points. Then $\mathcal{S}$ is tubular if and only if $\mathcal{S}$ satisfies the following three conditions.

(i) The homological quadratic form $\chi_{\mathcal{S}}$ is non-negative and has corank two.

(ii) $\chi_{\mathcal{S}}{ }^{-1}(1) \cap\left(\operatorname{rad} \chi_{\mathcal{S}}\right)^{\perp}=\emptyset$.

(iii) There exists a positive sincere radical vector of the Tits form $q_{\mathcal{S}}$.

Proof. Once again only the sufficiency requires an argument.

First, by Theorem A, $\mathcal{S}$ is derived tubular. Now since $\mathcal{S}$ is strongly simply connected, it has a postprojective component and therefore condition (iii) implies, that $\mathcal{S}$ is of infinite representation type [6]. Thus by [2], $\mathcal{S}$ is a branch-enlargement of a critical spectroid of extended Dynkin tubular type. If $\mathcal{S}$ is not tubular, there is exactly one critical convex subspectroid $\mathcal{R}$ of $\mathcal{S}$. But condition (iii) implies that $\operatorname{rad~q}_{A}$ is spanned by two critical (i.e. minimal) positive radical vectors $v_{1}$ and $v_{2}$. By [13], each of these vectors $v_{i}$ give rise to a critical convex subspectroid of $\mathcal{S}$, a contradiction showing the result.

4.3. It is not satisfactory to use two different quadratic forms in the formulation of the characterization in 4.2 (which, a posteriori, are in fact the same, since the global dimension is two). In fact, the homological quadratic form can be completely exchanged by the Tits form, but the orthogonality in condition (ii) remains with respect to the homological bilinear form, as shown in Theorem B. 
Proof of Theorem B. Again only the sufficiency has to be shown.

First observe that by condition (ii) and $2.2 \mathcal{S}$ does not contain a full and convex 2 -tubular subspectroid. Moreover since $q_{\mathcal{S}}$ is weakly non-negative, [18] implies that $\mathcal{S}$ is tame of polynomial growth. By (iii) and [13] we get that $\mathcal{S}$ is a critical or a tubular spectroid and hence by (i) the result.

4.4. Proof of Theorem C. By 2.3 and 4.3, we have to show that $\mathcal{S}$ is of polynomial growth if and only if qS is weakly non-negative and any full convex subspectroid $\mathcal{R}$ of $\mathcal{S}$ satisfying conditions (i) and (iii) of Theorem B, is a tubular spectroid. The result follows directly from [13].

\section{REFERENCES}

1. I. Assem and A. Skowroński, On some classes of simply connected algebras, Proc. London Math. Soc. (3), 56 (1988), 417-450. MR 89f:16023a

2. I. Assem and A. Skowroński, Algebras with cycle-finite derived categories, Math. Ann. 280 (1988), 441-463. MR 89f:16023b

3. I. Assem and A. Skowroński, Quadratic forms and iterated tilted Algebras, Journal of Algebra 128 (1990), 55-85. MR 91d:16020

4. M. Barot, Representation-finite derived tubular algebras, To appear.

5. R. Bautista, F. Larrión and L. Salmerón, On simply connected algebras, Journal of London Math. Soc. (2), 27 (1983), 212-220. MR 84i: 16028

6. K. Bongartz, Algebras and quadratic forms, J. London Math. Soc. 28 (1984), 461-469. MR 85i: 16036

7. K. Bongartz, Critical simply connected algebras, Manuscr. Math. 46 (1984), 117-136. MR 85j: 16026

8. O. Bretscher and P. Gabriel, The standard form of a representation-finite algebra, Bull. Soc. Math. France 111 (1983), 21-40. MR 85g:16014

9. P. Gabriel, B. Keller and A. V. Roiter, Representations of finite-dimensional algebras, Encycl. Math. Sc., 73 (1992), 1-176. MR 94h:16001b

10. D. Happel, On the derived category of a finite-dimensional algebra, Comment. Math. Helvetici 62 (1987), 339-389. MR 89c:16029

11. D. Hughes and J. Waschbüsch, Trivial extensions of tilted algebras, Proc. London Math. Soc. (3), 46 (1982), 346-364. MR 84m:16023

12. J. A. de la Peña, On the representation type of one point extensions of tame concealed algebras, Manusc. Math. 61 (1988), 183-194. MR 89h:16022

13. J. A. de la Peña, On the corank of the Tits form of a tame algebra, Journal of Pure and Applied Algebra 107 (1996), 89-105. MR 97h:16021

14. J. A. de la Peña, The Tits form of a Tame Algebra In: Representation Theory of Algebras, Can. Math. Soc. Conference Proc., 19 (1996), 159-183. MR 97j:16022

15. C. M. Ringel, Tame algebras and integral quadratic formsLecture Notes in Mathematics, Vol. 1099, Berlin, Heidelberg, New York, Springer, 1984. MR 87f:16027

16. A. Skowroński, Selfinjective algebras of polynomial growth, Math. Ann. 285 (1989), 177-199. MR 90k: 16024

17. A. Skowroński, Simply connected algebras and Hochschild cohomologies, Canadian Math. Soc., Conference Proceedings 14 (1993), 431-447. MR 94e:16016

18. A. Skowroński, Tame algebras with simply connected Galois coverings, Preprint, Poland (1996).

Instituto de Matemáticas, UNAM, 04510 México, D.F., México

E-mail address: barot@gauss.matem.unam.mx

E-mail address: jap@penelope.matem.unam.mx 